\title{
Control of tomato whiteflies using the confusion effect of plant odours
}

\author{
Colin R. Tosh $•$ Barry Brogan
}

Accepted: 26 February 2014 /Published online: 17 April 2014

(C) The Author(s) 2014. This article is published with open access at Springerlink.com

\begin{abstract}
Glasshouse tomato production is reduced by the whitefly Trialeurodes vaporariorum, a major worldwide pest of glasshouse crops. Whitefly control is achieved using pesticides or parasitoids, which have drawbacks. There is thus a need for other control methods. For instance, the use of volatile compounds from plant odours could profoundly change the behaviour of plant-eating insects. This practice should be safe because plant odours are usually harmless to humans. Therefore, we have studied the effect of plant volatiles on whiteflies feeding on tomato plants. The rationale was to use the confusion effect by supplying whiteflies with a super-abundance of volatiles. We removed plant headspace volatiles from multiple whitefly host plants. Then, we presented these volatiles to whiteflies feeding on tomato plants. At the same time, whitefly stylet penetration is monitored using electrical penetration graphs (EPG). Plant colonisation, egg laying and honeydew production are analysed in separate experiments. Data are compared to controls in which extracted volatiles from tomato and air alone are presented to the whiteflies. The only significant effect produced by exposure to multiple-host volatiles is a pronounced reduction in the incidence of phloem-related waveforms during the 15-h EPG recording. This represents a delay in, rather than a cessation of, phloem-related activities as there is no related reduction in long-term performance. The confusion effect thus does not appear to exert strong effects on whitefly behaviour here.
\end{abstract}

Keywords Trialeurodes vaporariorum · Tomato · Plant volatiles $\cdot$ Confusion $\cdot$ Pest $\cdot$ Insect

C. R. Tosh $(\bowtie) \cdot$ B. Brogan

School of Biology, Newcastle University, Ridley Building 2,

Newcastle upon Tyne NE1 7RU, UK

e-mail: colin.tosh@newcastle.ac.uk

\section{Introduction}

The glasshouse whitefly, Trialeurodes vaporariorum, is a major worldwide pest of glasshouse crops with control in the glasshouse usually achieved by chemical or biological (typically parasitoids) means (Fig. 1). Both control methods have their drawbacks, and here, we begin to investigate the possibility of supplementary control using harmless (to humans) plant volatiles that may nevertheless fundamentally alter the behaviour of insect plant pests.

Humans and other animals undertaking a task but supplied with an abundance of distracting information often become task-inefficient or 'confused'. In the field of predator-prey interactions, this has been termed the 'confusion effect' and has been used to explain why many prey animals live in groups (Milinski 1977; Krause and Ruxton 2002). In psychology, the positive relationship between reaction time to find a focal object and the number of items in the set containing the focal object has been used to derive influential models of visual search such as the Feature Integration Theory (Treisman and Gelade 1980; Wolfe et al. 2010). In entomology, this idea is embodied in the Neural Limitations Hypothesis (Dall and Cuthill 1997; Bernays 2001), stating that plant-eating insects specialise to increase the accuracy of plant location and selection behaviour.

So far, the 'confusion effect' and related ideas have been applied mainly to issues in fundamental biology, but what of its utility in applied biology? A few studies have investigated the relationship between plant species complexity and whitefly plant utilisation efficiency (Bernays 1999; Smith et al. 2001; Bird and Krüger 2007; Roff et al. 2012; Mansour et al. 2012), and most indicate that, at least for some performance parameters and host plant mixtures, plant diversity negatively impacts whiteflies. This conclusion reflects the wider relationship between plant species diversity and herbivore plant utilisation efficiency (reviewed in Risch 1983; 
a
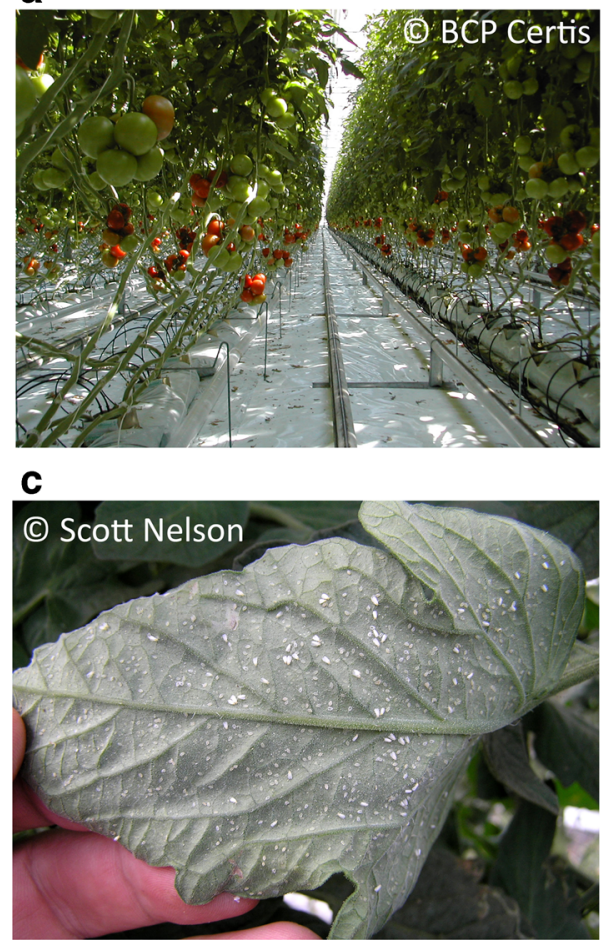

Fig. 1 Whiteflies and tomatoes: the nature of the problem. The commercial production of tomatoes can be a large-scale industrial process (a). Infestation of tomatoes growing in a glasshouse with a pest insect that eats tomato plants, and whose populations develop rapidly, can be disastrous. The glasshouse whitefly, Trialeurodes vaporariorum (b), is a major worldwide pest of tomato. It has long, thin mouthparts which it sticks into the plant tissue (b), and the mouthparts move through the tissue until they locate the phloem tissue from which they ingest. An infestation of b
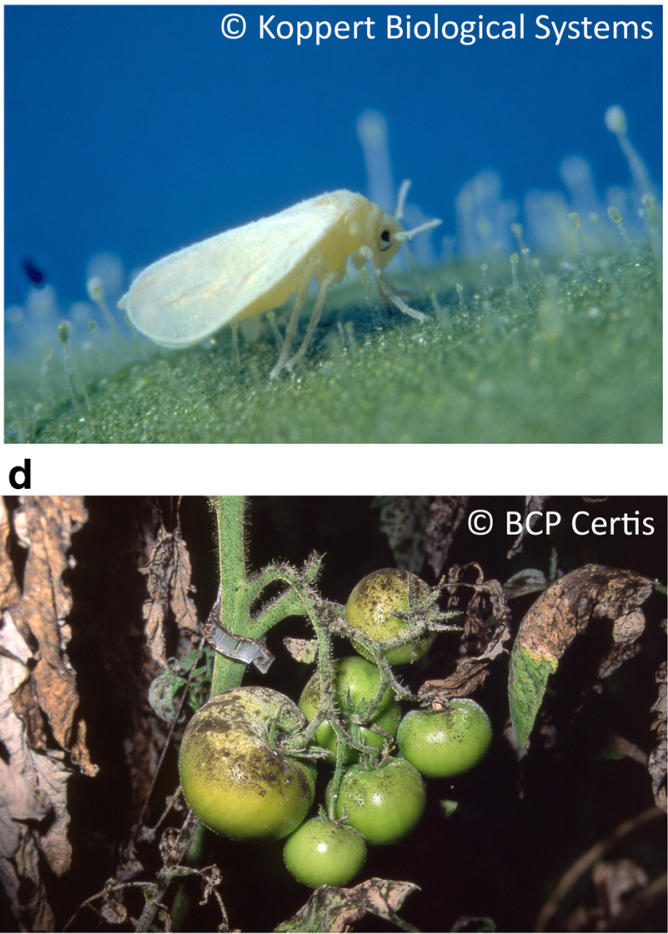

T. vaporariorum usually consists of adults (b and $\mathbf{c}$ ) and stationary 'plaque'-like larvae (c). Both adults and larvae ingest from the phloem and eject partially digested phloem sap from their rear end. This sweet, sticky honeydew coats surrounding vegetation and acts as a medium on which dark, sooty mould fungus can grow (d). The sooty mould problem is principally aesthetic, but if enough of it accumulates, it can reduce photosynthesis and affect yield. T. vaporariorum and other whitefly species can also spread plant viruses
Andow 1991; Stamps and Linit 1998; Schlapfer and Schmid 1999; Hooks and Johnson 2003; Barbosa et al. 2009; Letourneau et al. 2011; Cardinale et al. 2011; Agrawal et al. 2006). Most relevant to the present article is the study of Bernays (1999) because her study was specifically designed to investigate sensory confusion of whiteflies in relation to plant diversity (see also Bird and Krüger 2007). She studied the movement and fecundity of the whitefly Bemisia tabacion two sets of three host plants. Insects were observed on multiple plants of each of the three species and in a mixture of each. It was demonstrated that insects were significantly more 'restless' on plant mixtures than on any of the same-species plant groups. Effects on fecundity were less pronounced.

Here, we repeat elements of this study with the glasshouse whitefly, T. vaporariorum, including various refinements, adopted both to increase understanding of the precise mechanisms underlying confusion effects and with a long-term view to exploitation of observed effects within modern, large-scale horticultural systems. Firstly, by extracting plant volatiles from mixed species and moving them through an apparatus to feeding whiteflies, we concentrate on a single sense of the whitefly, olfaction, which is likely to be a key determinant of plant diversity effects on whiteflies. Secondly, as well as considering standard behavioural and life history metrics of performance (specifically here, short- and long-term settling rate, long-term honeydew production and long-term fecundity), we also subject volatile-exposed whiteflies simultaneously to an electrical penetration graph (EPG) recording of tissue penetration activities so the whole process of plant exploitation from settling through to long-term phloem utilisation can be studied in relation to plant volatile diversity. EPG (Tjallingii 1978) is a method in which a sap-sucking insect is attached to an extremely fine gold wire and a current passed through it. When the insect pierces the tissue, a circuit is completed, and the various subsequent behaviours of the mouthparts in the plant tissue can be recorded as distinct electrical waveforms. Lastly, while previous studies have generally studied the effects of plant mixtures on whitefly behaviour within these mixtures, if the confusion effect is to be used in modern horticultural systems, it will realistically have to be applied to plant monocultures. We therefore quantify all effects of mixed host volatiles on whiteflies feeding on a single host plant, tomato. While we believe the confusion effect is the most likely explanation for the modest negative 
effects of mixed host volatiles we observe here, as Bernays (1999), we are cautious in attributing them solely to the confusion effect and discuss other mechanisms that could contribute.

\section{Materials and methods}

\subsection{Whiteflies and plants}

Whiteflies, T. vaporariorum (Westwood), originated from a lab culture at Rothamsted Research which was first collected in 1960 in Kent on French bean and had subsequently been maintained in a large laboratory population. Prior to experiments, the culture had been maintained in CRTs lab for around a year as a population of several thousand individuals on preflowering aubergine (Solanum melongena) of the 'Moneymaker' variety (Marshalls Seeds Cat. 1020-2017) at $20{ }^{\circ} \mathrm{C}, 16: 8$ light/dark.

All plants used for culture, volatile collection, and EPGs were propagated approximately $60 \mathrm{~cm}$ away from a Harrier HR400SH 400W lamp housing a 400W Son-T bulb. Light was on a 16-h light, 8-h dark cycle in sync with the light regime that EPGs were conducted under. Temperature was $25^{\circ} \mathrm{C}$ during the light cycle and $20^{\circ} \mathrm{C}$ during the dark. All plants were grown from seed in Clover Multipurpose Compost (http://www.cloverpeat.co.uk/CLOVER-RETAILCOMPOST-1.html) in 9-cm-diameter and 8.7-cm-depth pots, one plant per pot (with the exception of watercress which was grown two to four plants per pot as plants are small), with liberal watering. Tomato (Lycopersicon esculentum Mill., 'Elegance', Cat. E/12/11, Batch 0113479253) was used preflowering with three to five fully emerged compound leafs. Cucumber, dwarf French bean and courgette were housed together (three plants of each; see Fig. $2 b$ for general arrangement) in the first of two volatile delivery experiments. Cucumber (Cucumis sativus, '(F1 hybrid) La Diva', Chiltern Seeds, Cat. 1359J) was pre-flowering with one fully emerged mature leaf at the time of placement into the volatile collection chamber. Dwarf French bean (Phaseolus vulgaris, 'Canadian Wonder', Chiltern Seeds, Cat 1816c) was pre-flowering with two fully emerged mature leafs at the time of placement into the volatile collection chamber. Courgette (Cucurbita pepo, 'Nero di Milano', Chiltern Seeds, Cat 1826) was preflowering with one fully emerged mature leaf at the time of placement into the volatile collection chamber. Watercress, watermelon and Savoy cabbage were housed together (three plants of each; see Fig. $2 b$ for general arrangement) in the second of two volatile delivery experiments. Watercress (Nasturtium officinale, 'Aqua', Sutton's Seeds, Cat. 161689) was pre-flowering with five to six fully emerged mature leafs at the time of placement into the volatile collection chamber. Watermelon (Citrullus lanatus, 'Red Star F1', Sutton's Seeds,
Cat. 171550) was pre-flowering with two to three fully emerged mature leafs at the time of placement into the volatile collection chamber. Savoy cabbage (Brasica oleracea, 'Ormskirk(1)-Rearguard', Sutton's Seeds, Cat.155962) was pre-flowering with two to three fully emerged mature leafs at the time of placement into the volatile collection chamber. Plants in both volatile delivery experiments were placed in the volatile collection chamber for approximately 2 weeks with liberal watering before being replaced by fresh plants. They were all pre-flowering at the time of removal. Plant species were all chosen as hosts of $T$. vaporariorum based on literature (Mound and Halsey 1978; Roditakis 1990; Gorman et al. 2002; Moreau and Isman 2011). The two control treatments consisted of nine pots with damp compost and nine pots each with a single tomato plant, placed in the volatile collection chamber.

\subsection{Volatile collection, volatile delivery, EPG, data analysis}

\subsubsection{Integrating volatile collection and delivery with EPG}

A standard eight-channel DC EPG system (Tjallingii 1978) was modified to allow the monitoring of whitefly feeding behaviour on tomato while supplying whiteflies with air containing volatiles from other host plants. Mixed-species plants or control pots were placed in a Perspex box measuring $\mathrm{L}$ $28 \mathrm{~cm} \times \mathrm{W} 28 \mathrm{~cm} \times \mathrm{H} 20 \mathrm{~cm}$ with four 2 -cm mesh-covered holes on one side and four outlet holes on the other (Fig. 2b). Outlet holes fitted 4-mm-ID fluorinated ethylene propylene (FEP) tubing (low odour emission) tapering through three Ysplitters to a single length of the same tubing, which connected the air pump. Distance from the outlets to the pump was $40 \mathrm{~cm}$. The volatile collection box was $50 \mathrm{~cm}$ away from two Osram L36W/840 bulbs on $16 \mathrm{~L} / 8 \mathrm{D}$ in synch with plant growth lamps and the main lights in the experimental room. The air pump was a Boxer 3000 gas pump with no sliding seals or components in the fluid path of the pump, ensuring contamination free transfer of media. It delivered volatileinfused air at a rate of $2.2 \mathrm{~L} / \mathrm{min}$ (Fig. 2b). Volatiles were pumped along a single length of 4-mm-ID FEP tube of length $312 \mathrm{~cm}$ to a bank of small FEP tubes and Y-splitters, splitting the flow into eight 4-mm-ID FEP tubes. The length of the Ysplitter bank was $21 \mathrm{~cm}$. The eight tubes, each $119 \mathrm{~cm}$ in length, fed into the Faraday cage where they joined the volatile delivery box. This was made of Perspex and measured L $79 \mathrm{~cm} \times \mathrm{W} 9 \mathrm{~cm} \times \mathrm{H} 6 \mathrm{~cm}$. Each inlet was covered with a $2-\mathrm{cm}$ diameter air deflector, with a 3-mm gap between the top of the deflector disk and the top of the box to allow inflow of air (Fig. 2c). Into the bottom of the box were cut eight $1.5-\mathrm{cm}$ diameter holes to allow the insertion of EPG probes, and into the back of the box, eight $1-\mathrm{cm}$ holes were cut to allow the insertion of tomato leaves. These $1-\mathrm{cm}$ holes were split along the middle, as was the whole box, so the box could be taken 


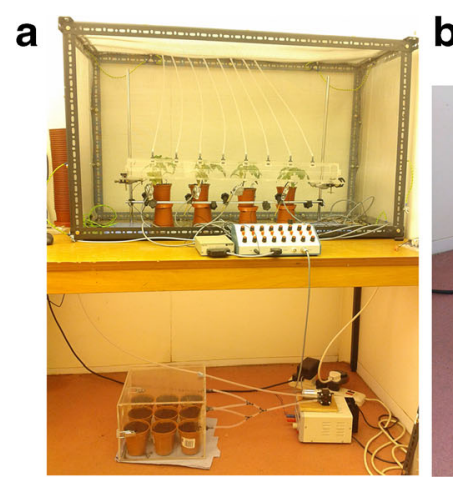

Fig. 2 The volatile collection, delivery and electrical penetration graph (EPG) apparatus. a the EPG and linked volatile delivery apparatus. The control treatment is shown where nine pots with compost only are inserted into the volatile collection chamber. $\mathbf{b}$ A more detailed picture of the volatile collection box shown in $\mathbf{a}$. The box is linked to an air pump and shown is the treatment in which volatiles from watercress, Savoy cabbage and melon are delivered to the electrically monitored whiteflies.
C

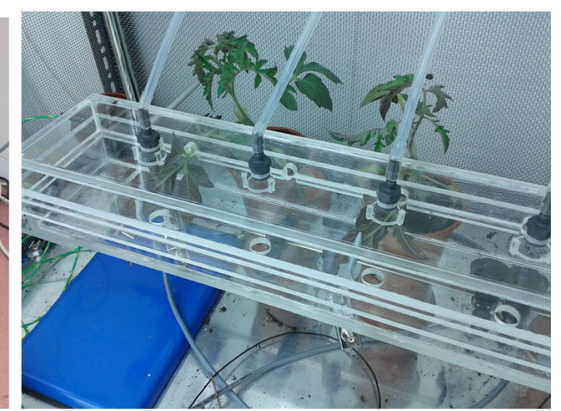

c A more detailed picture of the volatile delivery box shown in a. Volatiles from the collection box are input and deflected by deflector disks over the inlet holes. Holes at the back of the box allow insertion of tomato leaves, and holes at the bottom are used for insertion of EPG probes. The box splits in two along its length to allow insertion of tomato leaves without damaging the tissue apart and leaves inserted without damage to the plant tissue. We began to deliver volatiles to the tomato plants approximately $1 \mathrm{~h}$ before initiation of EPGs.

\subsubsection{EPG method}

The EPG method produces a waveform output, with different waveforms corresponding to defined feeding behaviour such as probing, xylem feeding and phloem feeding (Tjallingii 1978). We used an eight-channel DC EPG system supplied by EPG Systems (EPG-Systems, Dillenburg 12, 6703 CJ Wageningen, the Netherlands, http://www.epgsystems.eu/ contact.htm; Tjallingii 1978). We used the terminal leaflet of the uppermost, fully emerged compound leaf of the tomato plant. The terminal and next two leaflets were inserted into the volatile delivery chamber still attached to the main plant (Fig. 2c) and the terminal leaflet propped perpendicular to the base of the chamber with a length of Blu-Tack (Fig. 2c). While we would ideally have wished to run EPGs with the leaf positioned naturally and the whitefly feeding from the underside of it, it was found that the insect was unstable during EPGs in this position (probably due to the weight of the gold wire), and most fell from the plant during the 15-h recording, rendering traces unusable. Four plants and four channels were used in each daily experiment (Fig. 2a), and a different set of plants were used for EPGs in each daily run. Experiments were continued until we achieved 20 or so 15 -h recordings in each treatment. We generally recorded EPGs for around $20 \mathrm{~h}$, and a failed recording (discarded) was defined as one in which nonprobing (NP) began at some time before $15 \mathrm{~h}$ after the start of the recording and continued without additional waveforms until the end of the approximately 20 -h period. Although uncommon, traces that showed NP across the 15-h threshold but showed additional probing waveforms between 15 and $20 \mathrm{~h}$ were considered successful as it was assumed that the NP that occurred within the 15 -h period represented an insect still on the leaf 'choosing' not to probe. After placing the wired insects on the undersurface of the perpendicular leaf, when the EPG recording started, insects were closely monitored by the authors for 2 to $3 \mathrm{~h}$. Insects that fell from the leaf or became positioned on the leaf in a way that would not allow insertion of the mouthparts during this period were replaced on the leaf with a soft-tipped brush. After the 2-3-h period, the insects were left without intervention by the authors. The EPG electrode consisted of a $2-\mathrm{cm}$ nail, with a stiff but bendable $3-\mathrm{cm}$ length of copper wire soldered onto it and finally a $2-\mathrm{cm}$ length of $12.5-\mu \mathrm{m}$-diameter gold wire. We found it necessary to anesthetise these tiny, very mobile insects with $\mathrm{CO}_{2}$ to determine their sex and attach them via the dorsal thorax to the gold wire with water-based silver glue. Only females of mixed age taken at random from the stock culture were used for EPGs, and they were allowed at least $15 \mathrm{~min}$ of recovery time after anesthetization before being placed on the plant. All equipment within the Faraday cage was connected to the cage via earth wires attached at several points. Experiments were carried out under fluorescent light at $16 \mathrm{~h}$ light $/ 8 \mathrm{~h}$ dark, $20^{\circ} \mathrm{C}$, and EPGs were initiated between 6 and $9 \mathrm{~h}$ after the start of the light phase in the controlled environment (CE) room. Waveforms were identified using the waveform guide supplied with the Giga 4/8 EPG systems manual (http://www. epgsystems.eu/files/aphid\%20waveforms.pdf) as well as two studies investigating whitefly-specific waveforms (Lei and Tjallingii 1997; Lei et al. 1999). Output from the analysis of raw waveforms was converted into behavioural parameters using the spreadsheet outlined in Sarria et al. (2009).

Only female adults taken at random from the stock culture (mixed age) were monitored. The first experiment was a control in which nine pots filled with damp compost were placed in the volatile collection box (hereafter 'air control'). In the second control experiment, volatiles were delivered from 
nine pre-flowering tomato plants (hereafter 'tomato control'). This experiment controls for the 'quantity' of volatiles and is the control with which mixed host treatments (below) are compared. A difference between this control and mixed host treatments indicates that effects are due to quality and not the additional quantity of mixed host volatiles per se supplied to tomato leaves. In the third experiment, volatiles were delivered from three pre-flowering host plants of $T$. vaporariorum (Mound and Halsey 1978; Roditakis 1990; Gorman et al. 2002; Moreau and Isman 2011): cucumber, dwarf French bean and courgette (hereafter 'volatile mixture 1'). In the fourth experiment, volatiles were delivered from another three preflowering host plants of $T$. vaporariorum (Mound and Halsey 1978; Roditakis 1990; Gorman et al. 2002; Moreau and Isman 2011): watercress, watermelon and Savoy cabbage (hereafter 'volatile mixture 2'). The EPG recording lasted $15 \mathrm{~h}$.

\subsubsection{Data analysis}

Feeding parameters from EPGs were generally not normal, so were plotted as medians with confidence intervals for the median (Zar 1998) ( $n \approx 20)$. Within these data, proportional statistics were analysed using the chi-square test; otherwise, the Mann-Whitney $U$ test was used. Planned statistical comparisons undertaken were as follows: mixed host volatile treatments were compared with the tomato control and each other, and the tomato control was also compared to the air control.

2.3 Settling behaviour, long-term honeydew deposition, performance, data analysis

To study settling behaviour of unconstrained individuals, these four sets of experiments were essentially repeated, but EPG probes were removed, the delivery box was sealed to prevent escape of whiteflies, and 150 whiteflies of both sexes taken at random from stock culture (mixed age) were inserted into the delivery box with tomato leaves $(n=8)$ still attached to plants and observed every $30 \mathrm{~min}$ over the next $6 \mathrm{~h}$.

The volatile delivery chamber was set up as for EPGs except that eight tomato leafs were inserted into the chamber, and the chamber was made whitefly-tight using soft foam to plug petiole holes and fine gauze to cover other possible outlets. One hundred and fifty whiteflies of both sexes were taken at random from culture (hence mixed age) and split evenly between three 5-cm-diameter Petri dishes. Whiteflies in each of these dishes were anesthetised, and while still anesthetised, dishes were placed evenly spaced in the volatile delivery chamber, Petri dish lids were removed, and the chamber was sealed. Observations of the number of whiteflies on leafs were then undertaken every $30 \mathrm{~min}$ for $6 \mathrm{~h}$. A note of the number individuals on each leaf was taken after 24 and $54 \mathrm{~h}$, and the number of eggs on each leaf counted after $54 \mathrm{~h}$.
These procedures were repeated four times: once for the volatile mix 1 treatment (cucumber, dwarf French bean and courgette), once for the volatile mix 2 treatment (watercress, watermelon and Savoy cabbage) and once each for the controls. In each experiment, observations were begun 5-7 h after initiation of the light phase in the CE room. During settling experiments, we additionally placed aluminium foils of dimension $5 \times 7 \mathrm{~cm}$ under each leaf to collect honeydew. Foils were weighed before use in experiments and $54 \mathrm{~h}$ after initiation of the settling experiments.

The data on numbers of whiteflies per leaf vs. time were analysed by fitting a cubic model (whiteflies per leaf $=a+(b \times$ time $)+\left(c \times\right.$ time $\left.^{\wedge} 2\right)+\left(d \times\right.$ time $\left.^{\wedge} 3\right)$ and significant differences between settling parameters inferred from non-overlapping confidence intervals for model parameters. The 104 points (Fig. 3a) in each treatment were fitted using the LevenbergMarquart method with an SS convergence point of $1 \mathrm{E}-8$. Settling was also observed at 24 and $54 \mathrm{~h}$ and treatments compared using a small number of planned $t$-tests. The same planned comparisons as in EPG experiments above were undertaken. The number of eggs laid on each leaf after $54 \mathrm{~h}$ in settling experiments was analysed $(n=8)$, and again, the same planned comparisons as above undertaken using $t$-tests. The increase in weight of each foil $(n=8)$ was analysed using the same set of planned comparisons and $t$-tests.

\section{Results and discussion}

All the following statements are supported by statistics, which can be viewed in the legend of the appropriate figure.

\subsection{Settling experiments}

The confidence intervals of parameters of the cubic model fitted to represent settling rates of the whiteflies on tomato were overlapping for all of the comparisons made. Most fundamentally, there was no evidence that the form of the settling model differed between the tomato volatile control and either of the mixed volatile treatments (Fig. 3). This pattern was retained into longer-term settling patterns (24 and 54 h, Fig. 4). There was no significant difference in the number of whiteflies settling on tomato leafs over these longer periods between tomato volatile control and either of the mixed volatile treatments (Fig. 4).

\subsection{EPG parameters}

There was little difference between the tomato volatile control and either of the mixed host volatile treatments in many EPG plant penetration parameters (Fig. 5). The whiteflies showed the same median number of probes in the 15 -h recording period and displayed the same median time spent probing 
Number of whiteflies on leaf (eight leafs)

Air control

a

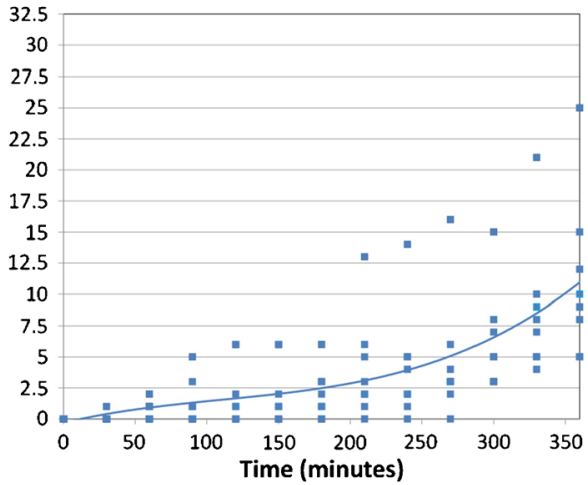

Number of whiteflies on leaf (eight leafs)

Mixed volatiles 1

C

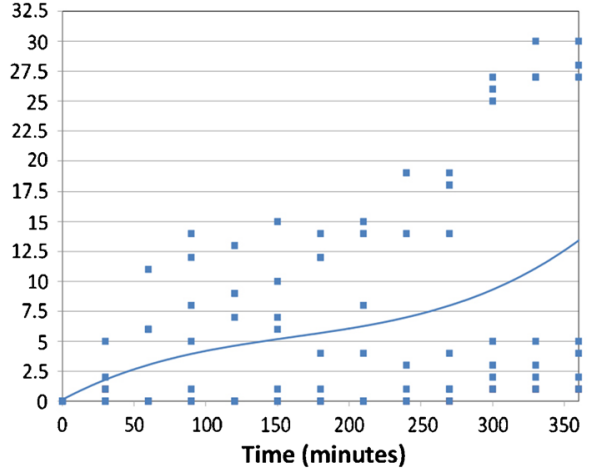

Parameter estimate with 95\% Cls

Model parameter a

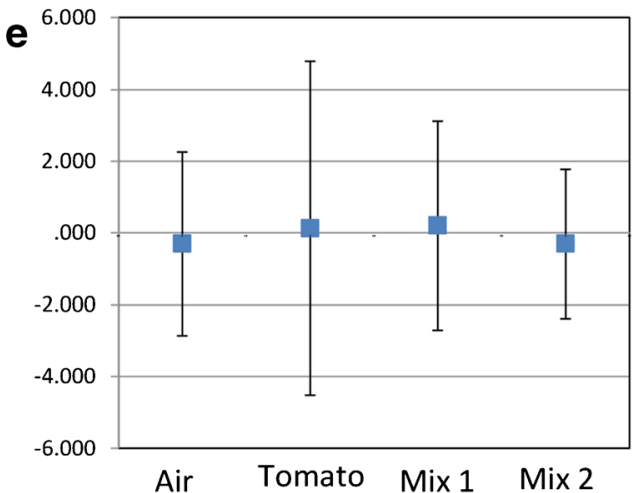

Parameter estimate with 95\% Cls

Model parameter c

g

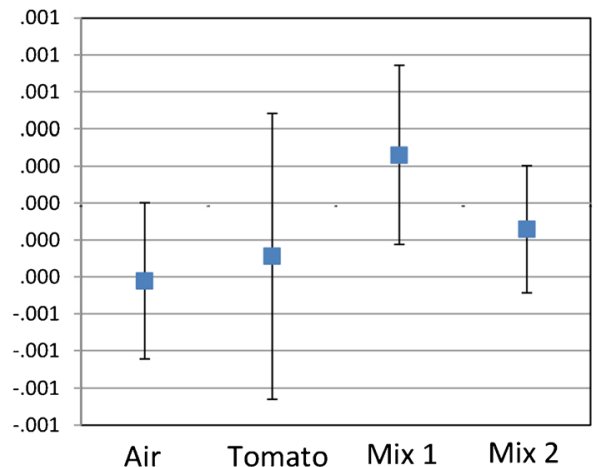

Number of whiteflies on leaf (eight leafs)

Tomato control

b

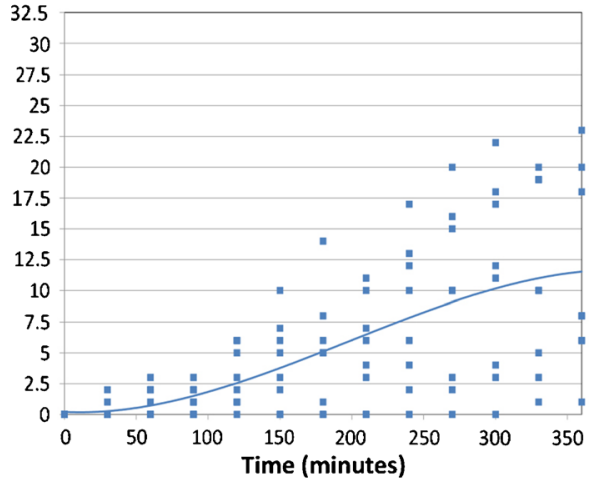

Number of whiteflies on leaf (eight leafs) Mixed volatiles 2

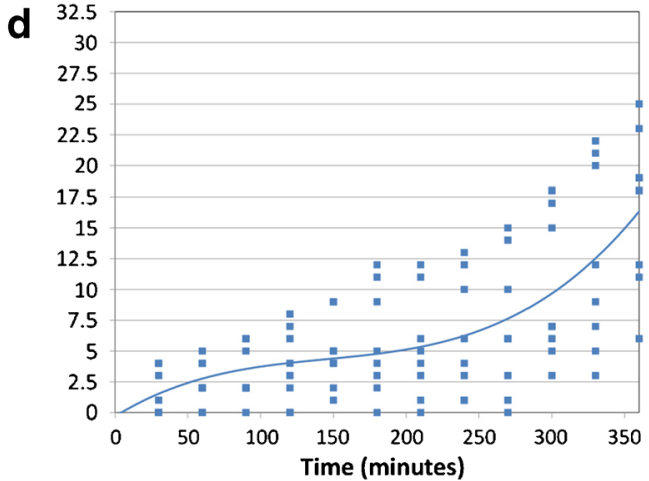

Parameter estimate with 95\% Cls

Model parameter $b$

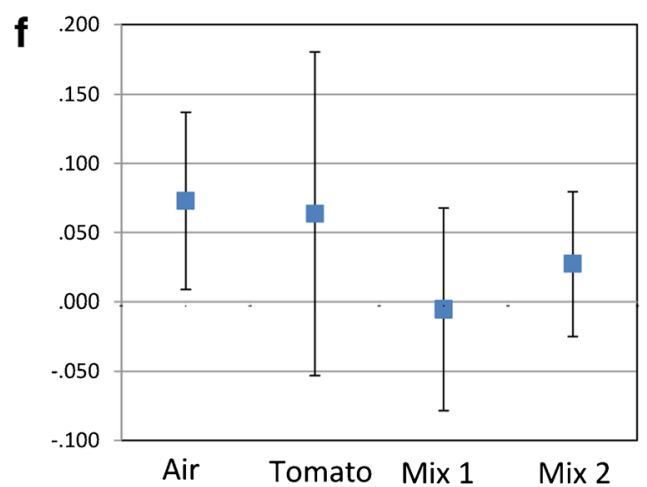

Parameter estimate with 95\% Cls

Model parameter $\mathrm{d}$

h

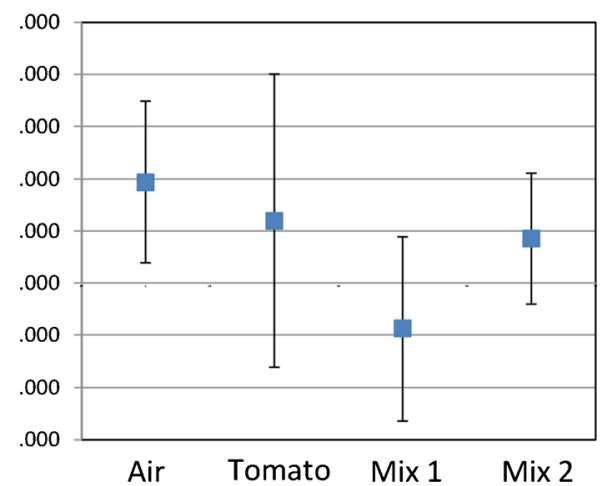


Fig. 3 Colonisation by whiteflies of tomato when they are exposed to air and air containing additional tomato and mixed alternative host plant volatiles. In a-d, a cubic model (line) of the type no. whiteflies $=a+(b \times$ time $)+\left(c \times\right.$ time $\left.^{\wedge} 2\right)+\left(d \times\right.$ time $\left.^{\wedge} 3\right)$ fitted to numbers of whitefly colonising eight tomato leafs across $6 \mathrm{~h}$ (squares). One hundred and fifty whiteflies of mixed sex were released to colonise leafs in each experiment. e to $\mathbf{g}$ Parameter estimates for the cubic model and $95 \%$ CIs. There is little evidence that mixed host plant volatiles reduce colonisation efficiency relative to a tomato volatile control, and supplying additional tomato volatiles has little effect on whitefly behaviour relative to a control of additional supply of air alone

the tissue in the 15-h period (Fig. 5a, b). There was, however, a dramatic difference in the incidence of the phloem utilisation waveforms E1 and E2 exhibited in the 15-h period. Around $70-80 \%$ of the individuals recorded in the air and tomato volatile controls exhibited phloem utilisation waveforms. Only around $20-30 \%$ of whiteflies in both mixed volatile treatments showed phloem utilisation waveforms in the same period. These effects are clearly visible (Fig. 5c).

\subsection{Long-term honeydew deposition and performance}

This effect of mixed host volatiles did, however, appear to represent a delay in normal phloem utilisation rather than a cessation of it as there were no corresponding effects on longer-term performance patterns such as honeydew deposition and egg laying. Fifty-four hours of honeydew deposition and egg laying did not differ significantly between the tomato volatile control and either of the mixed host volatile treatments (Fig. 6).

We have demonstrated that the principal effect of removing volatiles from mixed hosts of the glasshouse whitefly, T. vaporariorum, and presenting them to whiteflies feeding on another host, tomato, is to delay the initiation of phloem utilisation. This delay has little effect on plant colonisation or longer-term performance, so the confusion effect alone is unlikely to be a phenomenon that can be utilised effectively to control these organisms. We return to discuss the
Fig. 4 Colonisation by whiteflies of tomato when they are exposed to air and air containing additional tomato and mixed alternative host plant volatiles, after 24 and $54 \mathrm{~h}$, in the experiments summarised in Fig. 3. Within each graph, mixed host volatile treatments were compared statistically with the tomato control and each other, and the tomato control was also compared to the air control. Only significant statistical effects are shown, and absence of probability values indicates effects are insignificant at $\alpha=0.05 .24 \mathrm{~h}$ : TCMV1, $t_{(14)}=0.106, P=0.92$; TCMV2, $t_{(14)}=-0.366, P=0.72$; MV1-MV2, $t_{(14)}=-0.442$, $P=0.67$; AC-TC, $t_{(14)}=0.553$, $P=0.59 .52 h$ : TC-MV1, $t_{(14)}=0.324, P=0.75$; TC-MV2, $t_{(14)}=0.415, P=0.68 ;$ MV1-MV2, $t_{(14)}=0.029, P=0.98$; AC-TC, $t_{(14)}=0.147, P=0.89$
Whiteflies per leaf $(n=8, \pm 95 \% \mathrm{Cl}$ )

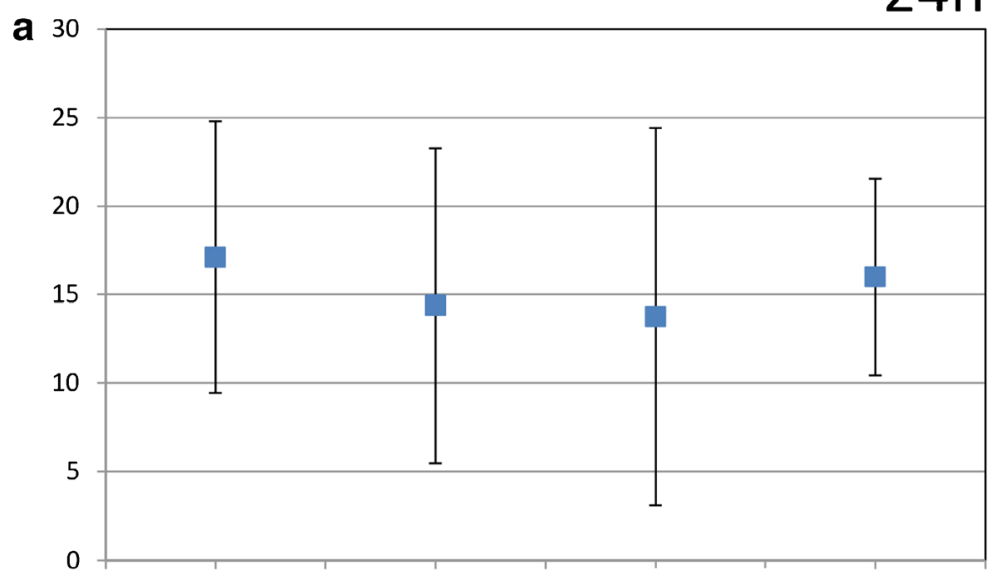

Air control Tomato control Mixed volatiles 1 Mixed volatiles 2

Whiteflies per leaf $(n=8, \pm 95 \% \mathrm{Cl})$

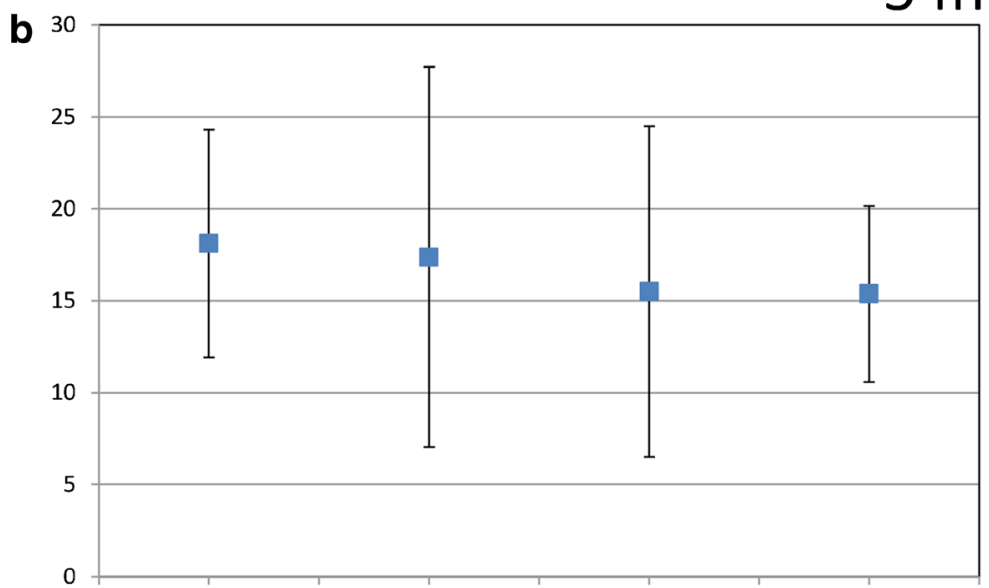

Air control Tomato control Mixed volatiles 1 Mixed volatiles 2 


\section{Median number}

of probes $(95 \% \mathrm{Cl}$

for median)
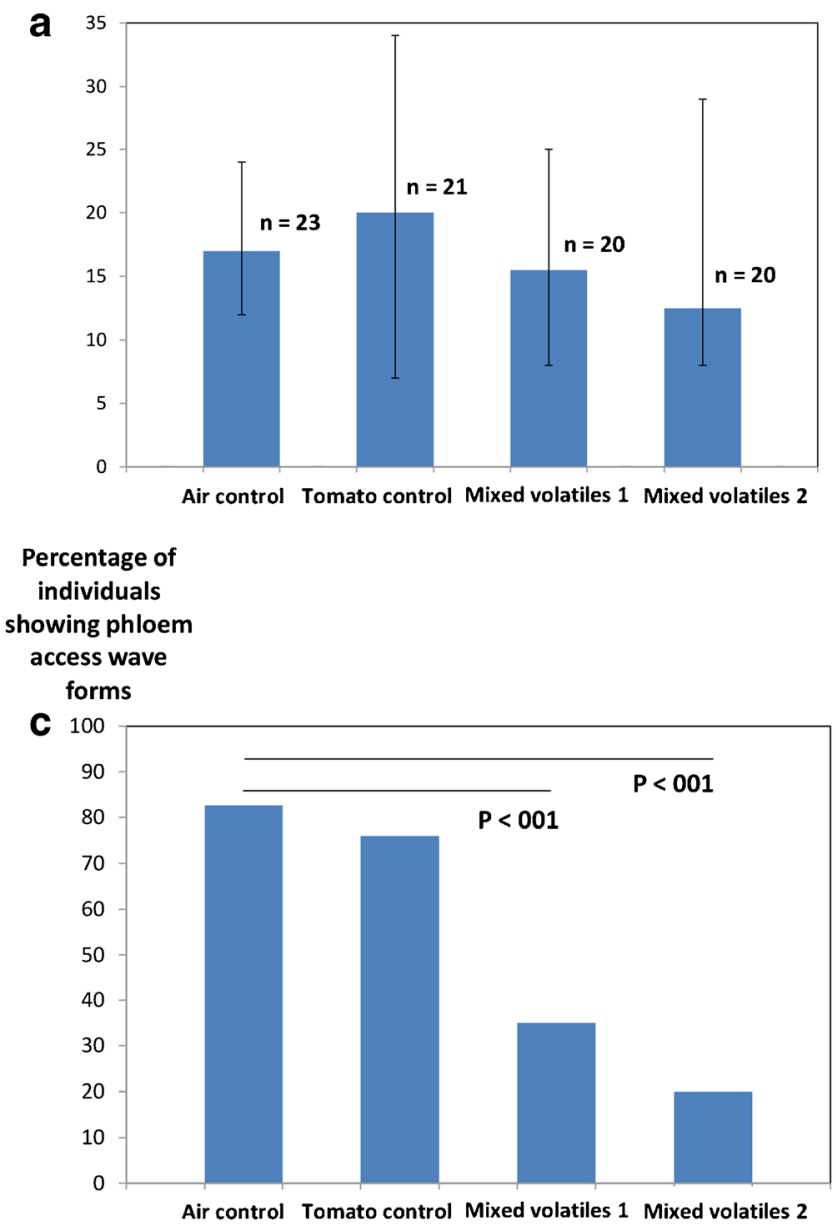

Fig. 5 Some parameters derived from electrical penetration graph (EPG) analysis of whiteflies feeding on tomato over $15 \mathrm{~h}$ while different host plant volatile mixtures were supplied to the insects. Within each graph, mixed host volatile treatments were compared statistically with the tomato control and each other, and the tomato control was also compared to the air control. Only significant statistical effects are shown, and absence of probability values indicates effects are insignificant at $\alpha=0.05$. a TC-

implications of these findings but firstly discuss determinants of the main significant negative effect we did demonstrate.

3.4 What is the cause of the delay in phloem utilisation?

We believe that the most likely explanation for the delay in phloem utilisation observed is the confusion effect. There is a vast literature showing that animals become inefficient at a task when simultaneously challenged with large quantities of relevant information (see 'Introduction'), and more specifically, previous studies (Bernays 1999; Bird and Krüger 2007) have implicated the confusion effect in the negative behavioural effect on whiteflies of host plant mixtures. By observing essentially the same effect when presenting volatiles from two sets of unrelated host plants relative to a pure tomato volatile

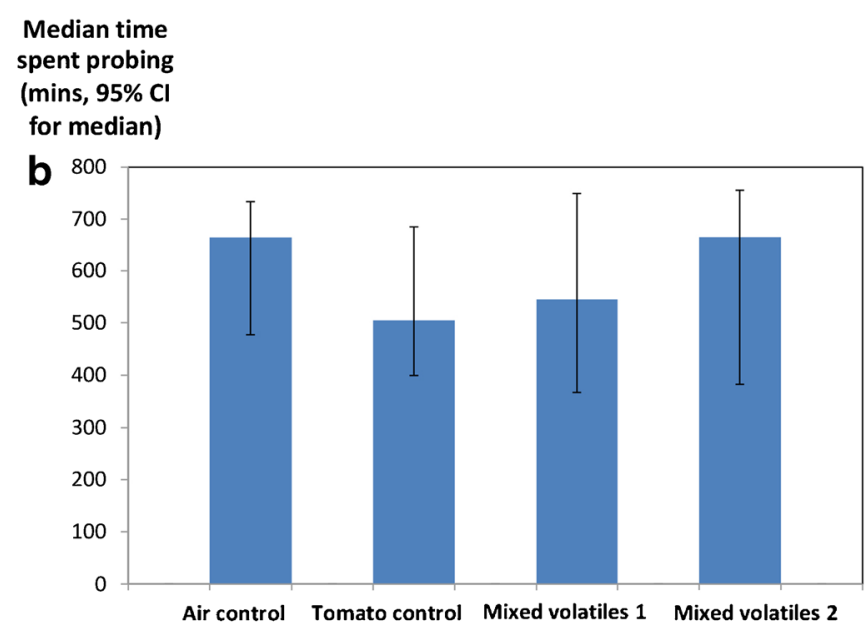

Air control Tomato control Mixed volatiles 1 Mixed volatiles 2

MV1, $U=197, P=0.73$; TC-MV2, $U=196, P=0.72$; MV1-MV2, $U=$ 199, $P=0.97$; AC-TC, $U=230, P=0.98$. b TC-MV1, $U=197, P=0.74$; TC-MV2, $U=178, P=0.40$; MV1-MV2, $U=182, P=0.64$; AC-TC, $U=$ 197, $P=0.41$. c TC-MV1, $X^{2}(1)=18.1, P=1.5 \mathrm{E}-05$; TC-MV2, $X^{2}(1)=$ $34.8, P=3.6 \mathrm{E}-09$; MV1-MV2, $X^{2}(1)=2.81, P=0.094$; AC-TC, $X^{2}(1)=$ $0.522, P=0.47$

control, we reason firstly that effects are not due to the increased quantity of volatiles supplied by the mixed host treatments but rather due to the quality of these volatile mixtures. Secondly, by observing exactly the same effect for two completely unrelated sets of mixed hosts, we reason that determinants of the effect are most likely to lie in a common characteristic of the mixtures: they represent an additional, complex source of information in a way that the pure tomato volatile control does not. The use of plants that whiteflies are known to use from the literature (Mound and Halsey 1978; Roditakis 1990; Gorman et al. 2002; Moreau and Isman 2011) for both sets of volatile extractions would also seem to exclude simple deterrence of feeding due to the extra volatiles. We did not ensure that the concentration of volatiles in the volatile presentation box exactly matched that in the mixed field plots, 
Fig. 6 Change in weight of aluminium foils placed under leafs in settling experiments (representing honeydew deposition, a) and egg lay per leaf (b), both after $54 \mathrm{~h}$. Within each graph, mixed host volatile treatments were compared statistically with the tomato control and each other, and the tomato control was also compared to the air control. Only significant statistical effects are shown, and absence of probability values indicates effects are insignificant at $\alpha=0.05$. a TC-MV1, $t_{(14)}=$ $0.710, P=0.49$; TC-MV2, $t_{(14)}=$ $0.753, P=0.46 ; \mathrm{MV} 1-\mathrm{MV} 2, t_{(14)}$ $=-0.277, P=0.79$; AC-TC, $t_{(14)}=$ $2.58, P=0.022$. b TC-MV $1, t_{(14)}=$ $0.802, P=0.44$; TC-MV2, $t_{(14)}=$ 1.45, $P=0.17$; MV1-MV2, $t_{(14)}=$ $0.736, P=0.47$; AC-TC, $t_{(14)}=$ $-0.029, P=0.98$

\section{$54 \mathrm{~h}$ change in foil weight \\ (mg, $\pm 95 \% \mathrm{Cl}, \mathrm{n}=8$ )}

a

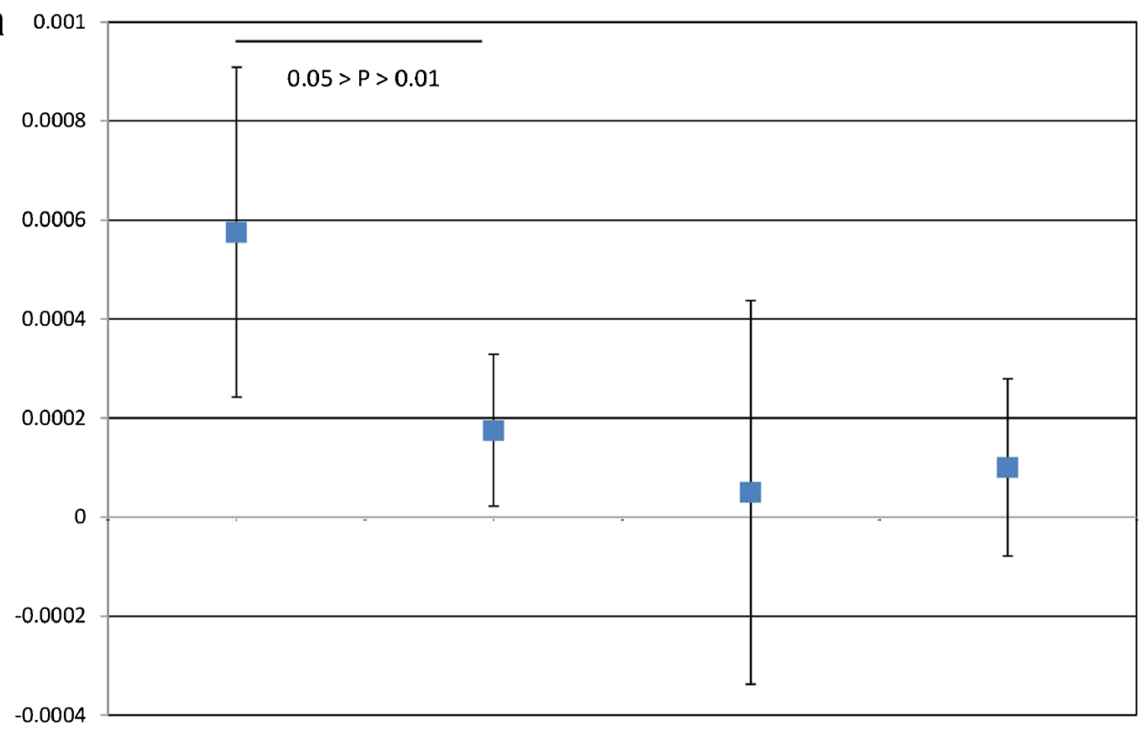

Air control

Tomato control Mixed volatiles 1 Mixed volatiles 2

\section{$54 \mathrm{~h}$ egg lay $( \pm 95 \% \mathrm{Cl}, \mathrm{n}=8$ )}

b

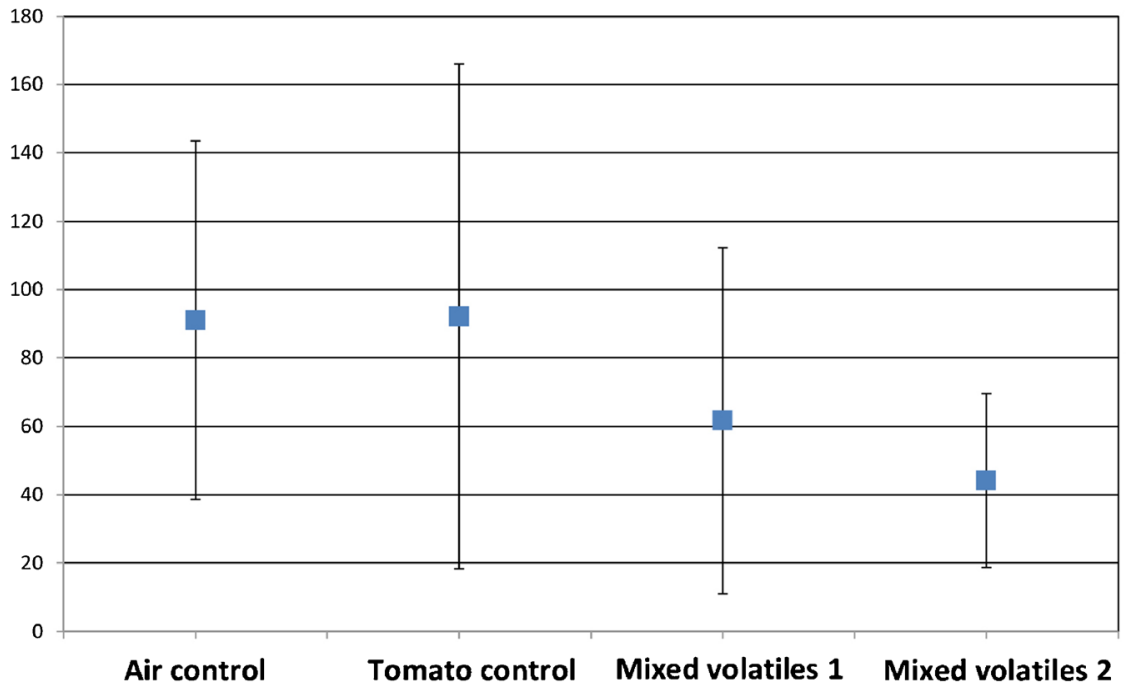

and host volatile components presented at an unnatural concentration could conceivably induce deterrence. This scenario would, however, require by chance that this effect occurred in both mixed host treatments and not in the pure tomato volatile treatment, a possibility, but a less parsimonious scenario than that of induction through informational complexity. The running of a tomato volatile control excludes the possibility that effects are caused by habituation of insects to a putative ubiquitous volatile that stimulates phloem seeking. The possibility of alternative host plants inducing a defensive response in tomato is unlikely as this usually requires that the emitting plants are subject to herbivore attack (Heil and Ton 2008; Dicke and Baldwin 2010). It should not automatically be assumed that infestation is required to prime a defensive response in neighbouring plants, however, as barley exposed to thistle for 5 days can be less suitable for aphid utilisation (Glinwood et al. 2004). Even supposing that a volatile component presented could induce such an effect, this would again require that these effects were by chance expressed in mixed host treatments but not in tomato. The process of phloem location is proposed to involve a pre-programmed radial orientation of stylet progression via cell walls with regular punctures into cells along the pathway in which sucrose concentration and $\mathrm{pH}$ are assessed, arresting or inducing further stylet progression (Hewer et al. 2010, 2011). It may be that the assessment of sucrose and $\mathrm{pH}$ information is interrupted by the constant bombardment of the olfactory organs by multiple positive, relevant stimuli, much in the same way that a 
teacher's marking could be compromised if a loud television is turned on in the same room. The most plausible alternative possibility is that the effects we observed could be due to a breakdown of multimodal integration where taste conflicts with odour information (see Roach et al. 2006 and refs therein). We have assumed there will be no conflict in the insects as both taste and olfactory cues are 'positive', but if sensory conflict occurs in the insect unless there is a degree of chemical correspondence between taste and smell cues, then this mechanism is possible.

3.5 A research programme to develop plant odour-based methods to control glasshouse whiteflies

We are currently investigating the potential for odour-based methods of control against glasshouse whiteflies. The present paper indicates that increasing odour complexity per se is not likely to provide an effective means on its own; however, increasing odour complexity did have some negative consequences for whitefly plant use here, so it could be used in conjunction with more potent effects. Moreover, we have not taken into account the need for whiteflies to orientate towards plants, and it is quite possible that this aspect of plant utilisation could be affected by sensory confusion (Bernays and Funk 1999). It should be noted that making use of the mild effects demonstrated here would first require that they are not subject to habituation and, if they are, determining what could be done to overcome this. Numerous studies indicate that non-host plant volatiles can have negative performance consequences for pest insects either through repellence or masking of host odours (Thiery and Visser 1986; Regnault-Roger 1997; Foster and Harris 1997; Ngoh et al. 1998; Isman 2006; Szendrei and Rodriguez-Saona 2010; Togni et al. 2010). Imagine a biological model for whitefly control consisting of one non-host plant that omits repellent volatiles, one non-host that emits odour-masking volatiles and a tomato host that is infested with whiteflies. Volatiles from such a model might reduce whitefly performance on tomato through not only repellence, odour masking and induction of host plant defences, but also through mild confusion effects due to the overall complexity of the volatile mixture. This model has the potential to strongly impact whitefly performance and to demonstrate good defence against resistance due to its multimechanistic nature, and it is the model we are at present developing. Of course, even having established a successful biological model, the fundamental causes of effects must be determined and distilled into a cheap, easily implemented system that can be applied to glasshouse monoculture, so this research programme is still in its infancy.

Acknowledgments This work was funded by the UK Natural Environment Research Council (NERC) grant NE/H015469/1.
Open Access This article is distributed under the terms of the Creative Commons Attribution License which permits any use, distribution, and reproduction in any medium, provided the original author(s) and the source are credited.

\section{References}

Agrawal AA, Lau JA, Hamba PA (2006) Community heterogeneity and the evolution of interactions between plants and insect herbivores. Q Rev Biol 81:349-376

Andow DA (1991) Vegetational diversity and arthropod population response. Annu Rev Entomol 36:561-586

Barbosa P, Hines J, Kaplan I et al (2009) Associational resistance and associational susceptibility: having right or wrong neighbors. Annu Rev Ecol Evol Syst 40:1-20. doi:10.1146/annurev.ecolsys.110308. 120242

Bernays E (1999) When host choice is a problem for a generalist herbivore: experiments with the whitefly, Bemisia tabaci. Ecol Entomol 24:260-267

Bernays EA (2001) Neural limitations in phytophagous insects: implications for diet breadth. Annu Rev Entomol 46:703-727

Bernays EA, Funk DJ (1999) Specialists make faster decisions than generalists: experiments with aphids. Proc Biol Sci 266:151-156

Bird TL, Krüger K (2007) Response of the polyphagous whitefly Bemisia tabaci B-biotype (Hemiptera: Aleyrodidae) to crop diversification - influence of multiple sensory stimuli on activity and fecundity. Bull Entomol Res 96:15-23. doi:10. 1079/BER2005398

Cardinale BJ, Matulich KL, Hooper DU et al (2011) The functional role of producer diversity in ecosystems. Am J Bot 98:572-592. doi:10. 3732/ajb. 1000364

Dall S, Cuthill I (1997) The information costs of generalism. Oikos 80: 197-202

Dicke M, Baldwin IT (2010) The evolutionary context for herbivoreinduced plant volatiles: beyond the "cry for help". Trends Plant Sci 15:167-175. doi:10.1016/j.tplants.2009.12.002

Foster SP, Harris MO (1997) Behavioral manipulation methods for insect pest-management. Annu Rev Entomol 42:123-146. doi:10.1146/ annurev.ento.42.1.123

Glinwood R, Ninkovic V, Pettersson J, Ahmed E (2004) Barley exposed to aerial allelopathy from thistles (Cirsium spp.) becomes less acceptable to aphids. Ecol Entomol 29:188-195. doi:10.1111/j.03076946.2004.00582.x

Gorman K, Hewitt F, Denholm I, Devine GJ (2002) New developments in insecticide resistance in the glasshouse whitefly (Trialeurodes vaporariorum) and the two-spotted spider mite (Tetranychus urticae) in the UK. Pest Manag Sci 58:123130. doi:10.1002/ps.427

Heil M, Ton J (2008) Long-distance signalling in plant defence. Trends Plant Sci 13:264-272. doi:10.1016/j.tplants.2008.03.005

Hewer A, Will T, van Bel AJE (2010) Plant cues for aphid navigation in vascular tissues. J Exp Biol 213:4030-4042. doi:10.1242/jeb.046326

Hewer A, Becker A, van Bel AJE (2011) An aphid's Odyssey - the cortical quest for the vascular bundle. J Exp Biol 214:3868-3879. doi: $10.1242 / \mathrm{jeb} .060913$

Hooks CRR, Johnson MW (2003) Impact of agricultural diversification on the insect community of cruciferous crops. Crop Prot 22:223238. doi:10.1016/S0261-2194(02)00172-2

Isman MB (2006) Botanical insecticides, deterrents, and repellents in modern agriculture and an increasingly regulated world. Annu Rev Entomol 51:45-66. doi:10.1146/annurev.ento.51.110104.151146

Krause J, Ruxton GD (2002) Living in groups. Oxford University Press, USA 
Lei H, Tjallingii W (1997) Effect of tethering during EPG recorded probing by adults of the greenhouse whitefly. J Appl Entomol 121:211-217

Lei H, Lenteren JC, Tjallingii WF (1999) Analysis of resistance in tomato and sweet pepper against the greenhouse whitefly using electrically monitored and visually observed probing and feeding behaviour. Entomol Exp Appl 92:299-309. doi:10.1046/j.15707458.1999.00551.x

Letourneau DK, Armbrecht I, Rivera BS et al (2011) Does plant diversity benefit agroecosystems? A synthetic review. Ecol Appl 21:9-21

Mansour SAA, Roff MNM, Saad KA et al (2012) Responses of whitefly, Bemisia tabaci (Genn.) (Homoptera: Aleyrodidae) population on tomato Lycopersicon esculentnm mixed with other crops under glasshouse conditions. APCBEE Procedia 4(4):48-52. doi:10. 1016/j.apcbee.2012.11.009

Milinski M (1977) Experiments on the selection by predators against spatial oddity of their prey 1 . Z Tierpsychol 43:311-325

Moreau TL, Isman MB (2011) Trapping whiteflies? A comparison of greenhouse whitefly (Trialeurodes vaporariorum) responses to trap crops and yellow sticky traps. Pest Manag Sci 67:408-413. doi:10. 1002/ps.2078

Mound L, Halsey S (1978) Whitefly of the world. British Museum Natural History. John Wiley, Chichester

Ngoh SP, Choo LEW, Pang FY et al (1998) Insecticidal and repellent properties of nine volatile constituents of essential oils against the American cockroach, Periplaneta americana (L.). Pestic Sci 54: 261-268. doi:10.1002/(SICI)1096-9063(1998110)54:3<261::AIDPS794>3.0.CO;2-C

Regnault-Roger C (1997) The potential of botanical essential oils for insect pest control. Integr Pest Manag Rev 34:25-34

Risch SJ (1983) Intercropping as cultural pest control: prospects and limitations. Environ Manag 7:9-14

Roach NW, Heron J, McGraw PV (2006) Resolving multisensory conflict: a strategy for balancing the costs and benefits of audio-visual integration. Proc Biol Sci 273:2159-2168. doi:10.1098/rspb.2006. 3578
Roditakis N (1990) Host plants of greenhouse whitefly Trialeurodes vaporariorum Westwood (Homoptera: Aleyrodidae) in Crete. Weeds 37:217-224

Roff MNM, Saad KA, Hanifah YM et al (2012) Effect of several vegetable combinations on the population of Bemisia tabaci (Homoptera: Aleyrodidae) under glasshouse conditions. Acad J Entomol 5:169-173. doi:10.5829/idosi.aje.2012.5.3.66209

Sarria E, Cid M, Garzo E, Fereres A (2009) Excel Workbook for automatic parameter calculation of EPG data. Comput Electron Agric 67:35-42. doi:10.1016/j.compag.2009.02.006

Schlapfer F, Schmid B (1999) Ecosystem effects of biodiversity: a classification of hypotheses and exploration of empirical results. Ecol Appl 9:893-912

Smith HA, Sorley RMC, Arnoldo J, Izaguirre S (2001) Effect of intercropping common bean with poor hosts and nonhosts on numbers of immature whiteflies (Homoptera: Aleyrodidae) in the Salama Valley, Guatemala. Environ Entomol 30:89-100

Stamps WT, Linit MJ (1998) Plant diversity and arthropod communities: implications for temperate agroforestry. Agrofor Syst 39:73-89

Szendrei Z, Rodriguez-Saona C (2010) A meta-analysis of insect pest behavioral manipulation with plant volatiles. Entomol Exp Appl 134:201-210. doi:10.1111/j.1570-7458.2009.00954.x

Thiery D, Visser JH (1986) Masking of host plant odour in the olfactory orientation of the Colorado potato beetle. Entomol Exp Appl 41: 165-172. doi:10.1111/j.1570-7458.1986.tb00524.x

Tjallingii WF (1978) Electronic recording of penetration behaviour by aphids. Entomol Exp Appl 24:721-730

Togni PHB, Laumann RA, Medeiros MA, Sujii ER (2010) Odour masking of tomato volatiles by coriander volatiles in host plant selection of Bemisia tabaci biotype B. Entomol Exp Appl 136: 164-173. doi:10.1111/j.1570-7458.2010.01010.x

Treisman A, Gelade G (1980) A feature integration theory of attention. Cogn Psychol 12:98-136

Wolfe JM, Palmer EM, Horowitz TS (2010) Reaction time distributions constrain models of visual search. Vis Res 50:1304-1311. doi:10. 1016/j.visres.2009.11.002

Zar JH (1998) Biostatistical analysis. Prentice Hall, Upper Saddle River 